\title{
Is the use of growth hormone and melatonin justified in spinal cord injuries?
}

\begin{abstract}
In this review we analyzed the possibility that tetraplegic patients suffer a deficient $\mathrm{GH}$ secretion occurring as a consequence of the loss of adrenergic control of somatostatin release, the inhibitor of pituitary GH synthesis and release. A deficient GH secretion can contribute to enhance the metabolic abnormalities seen in tetraplegics, increase the cardiovascular risk and osteopenia/osteoporosis. We also analyzed why orthostatic hypotension exists in tetraplegics, because of the loss of sympathetic afferences to the cardiovascular system. In addition we examined why the pineal production of melatonin is absent in tetraplegics, as a result of the loss of adrenergic signals from the upper cervical ganglion to the pineal gland; this results in sleep disorders and poor quality of life, but also in increased oxidative stress and the loss of the multiple beneficious effects that melatonin plays in the human body (oncostatic, anti-inflammatory, neuroprotective, etc). Lastly we describe a case of exaggerated allergic response to an allergen in a young tetraplegic girl. We conclude that because of the decreased production of the natural inhibitors of histamine (adrenaline and ACTH-independent corticosteroids), tetraplegic patients may have a special enhanced sensitivity to allergens that should be studied. In summary, GH and Melatonin are useful and safe in the treatment of tetraplegic patients.
\end{abstract}

Keywords: growth hormone, melatonin, spinal cord injury, sympathetic activity, orthostatic hypotension, dyslipidemia, superior cervical ganglion, pineal gland, insulin-like growth factor 1
Volume 4 Issue 2 - 2017

\author{
Jesús Devesa, ${ }^{1}$ Natalia López Segade, ${ }^{2}$ \\ Jacobolsorna, ${ }^{3}$ Pablo Devesa, ${ }^{4}$ Sara \\ Castellanos, ${ }^{2}$ Carlos I Puell ${ }^{5}$ \\ IScientific Direction, Medical Center Foltra, Spain \\ ${ }^{2}$ ChildrenPhysiotherapy, Medical Center Foltra, Spain \\ ${ }^{3}$ Infirmary, Medical Center Foltra, Spain \\ ${ }^{4}$ Research and Development, Medical Center Foltra, Spain \\ ${ }^{5}$ Physical Medicine and Rehabilitation, Medical Center Foltra, \\ Spain
}

Correspondence: Jesús Devesa, Scientific Direction, Medical Center Foltra, Travesía de Montouto 24, 15886

Teo, Spain, Tel +34 981802928,

Email jesus.devesa@usc.es, devesa.jesus@gmail.com

Received: July 30, 2017 | Published: August 28, 2017
Abbreviations: GH, growth hormone; MT, melatonin; SCI, spinal cord injury; IGF1, insulin-like growth factor 1; LDL, lowdensity lipoprotein; HDL, high-density lipoprotein; SS, somatostatin; GHRH, growth hormone releasing hormone; AIS, american spinal injury association impairment scale

\section{Introduction}

Spinal cord injury ( $\mathrm{SCI}$ ) is a devastating injury that leads to the total or partial loss of motor and sensory functions, but also affects the function of many body systems producing an almost total dependence and poor quality of life, ${ }^{1}$ although the level of the spinal cord in which the injury occurs and the degree of the injury are strong determinants of morbidity associated with the lesion. According to data provided by WHO in 2013, about 500.000 people worldwide suffer a SCI every year, ${ }^{2}$ most of them because of a spinal cord trauma, although vascular infarctions of the vertebral arteries are also a common cause. Most of the studies performed in patients with SCI analyze cardiovascular risk, due to increased plasma LDL cholesterol and decreased HDL cholesterol, ${ }^{3-7}$ increased obesity and oral carbohydrate intolerance and insulin resistance which leads to a high prevalence of diabetes mellitus in SCI; ${ }^{5,8}$ other studies in SCI analyze the degree of osteoporosis, which our group observed in $88.4 \%$ of the 43 patients before being treated at our Medical Center, ${ }^{9}$ resulting in a high risk of fractures of the head of the femur when receiving physiotherapy or associated with robotic treadmill training. Since 1978, many other studies analyze the existence of orthostatic hypotension in SCI patients, ${ }^{10-12}$ although the appearance of this low blood pressure depends on the level of the spinal cord in which the injury occurred. This orthostatic hypotension mainly occurs in SCI patients with injuries above T6 (Figure $1 \&$ Figure 2), because of the impaired sympathetic control of the cardiovascular system, and is higher in patients with cervical lesions in which plasma levels of catecholamines are lower than in thoracic SCI patients. ${ }^{11}$

Surprisingly and despite that most of the metabolic affectations occurring in SCI patients, above described, have also been reported in patients with deficient GH secretion, only two ancient studies describe the existence of GH-deficiency in $\mathrm{SCI},{ }^{13,14}$ a finding also observed by our group. ${ }^{9}$ These studies ${ }^{13,14}$ attributed the lack of significant $\mathrm{GH}$ secretion or its poor response to intravenous arginine administration to the fact that SCI patients cannot perform daily vigorous exercise and their life is rather sedentary. However, while it is true that exercise is a powerful stimulus for inducing pituitary GH release, its effects are mediated by the exercise-dependent hypothalamic production of catecholamines therefore leading to an adrenergic inhibition on SS release and the consequent stimulation of hypothalamic GHRH discharge into the portal blood vessels. ${ }^{15}$

As it happens with GH and SCI, the circadian rhythms of MT have less well studied in human SCI patients. Classically, it is well known that the last pathway for production of MT by the pineal gland is of adrenergic nature. The first study which analyzed diurnal rhythms of this involved analysis of indolamine in patients with SCI. This study was carried out in 1989 and reported that the cervical region of the spinal cord is essential for the normal circadian secretion of pineal MT in humans. ${ }^{16}$ This was confirmed almost tenyears later, ${ }^{17}$ in a study carried out in three tetraplegic patients with injury to their cervical spinal cord and two paraplegics in which the injury affected the upper thoracic spinal cord (complete spinal cord section in both types of patients). While the secretion of MT was completely lost in tetraplegic SCI, plasma levels of the hormone were of normal amplitude and showed a normal rhythmicity in the paraplegic patients. ${ }^{17}$ 
Even less known is the possibility that SCI patients in which injuries affect the upper thoracic and cervical regions of the spinal cord may suffer an enhanced sensitivity in response to allergens. Although it is not the aim of this review, an allergic response leads to the release of histamine from mast cells and basophils. Depending on the severity of the allergic attack it may produce catastrophic consequences to the patient, even his/her death. In 1994 it was reported that histamine, via $\mathrm{H} 1$ receptor, induced the local release of adrenaline in the rat adrenal gland, ${ }^{18}$ which, in turn, stimulated corticosterone release. It is not known whether histamine also induces the release of hypothalamic catecholamines, both in rats and humans, although there are data indicating that histamine induces hypothalamic paraventricular release of oxytocin by stimulating the release of noradrenaline. ${ }^{19}$ In any case, the administration of adrenaline is the first choice against an anaphylactic reaction. Therefore, the possibility exists that SCI affecting the efferent adrenergic pathways to the adrenal gland and/ or hypothalamus may represent a latent danger for SCI patients who suffer an allergic reaction.

\section{Discussion}

We first described that the pituitary secretion of $\mathrm{GH}$ is mainly dependent on the inhibitory role that alpha-2-adrenergic pathways exert on hypothalamic SS release. ${ }^{15,20-24}$ In turn, $\beta$-adrenergic responsiveness, which depends on a low supply of catecholamines to SS-producing neurons, enhances SS secretion into hypothalamichypophyseal portal vessels produces the blockade of the pituitary release of GH. ${ }^{25}$ On these bases it is feasible to assume that complete (AIS A) SCI injuries above T5-T6 will lead to the loss of the afferent inputs from the spinal cord to the sympathetic ganglionic chain (Figure $1 \&$ Figure 2) resulting in decreased or an absence of the supply of catecholamines to the hypothalamus. The result of this situation would be an increased SS release and, consequently, deficient or insufficient GH secretion. In fact, in our previous study ${ }^{9}$ we found that in tetraplegic patients the $\mathrm{GH}$ peak elicited by oral administration of clonidine $\left(0,150 \mathrm{mg} / \mathrm{m}^{2}\right.$, Catapresán, BoehringerIngelheimEspaña, Barcelona, España) was under normal values.

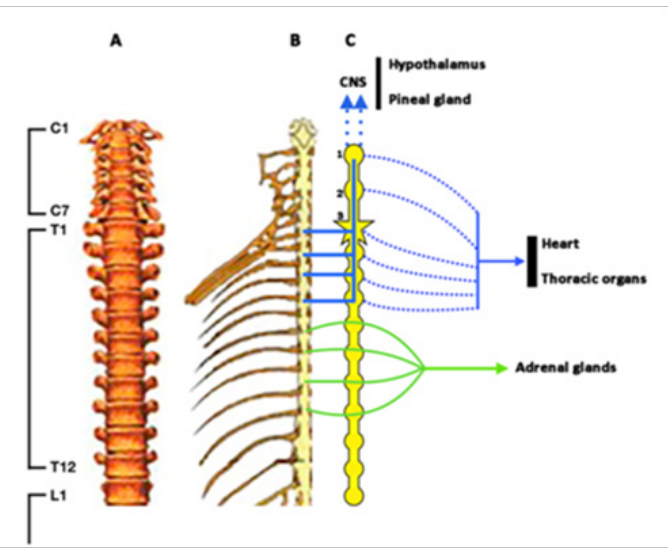

Figure I Schematic representation of the spinal column until LI (A), and the spinal cord (B) and its afferences to the sympathetic ganglionic chain (C) CNS: Central nervous system. I, Upper cervical ganglion. 2, Middle cervical ganglion. 3, Lower cervical ganglion or star-shaped ganglion. Blue lines indicate afferent signals from the spinal cord to the sympathetic ganglionic chain and from it to the Upper cervical ganglion. Blue dotted lines ending in arrow indicate sympathetic efferences to the CNS, particularly to the hypothalamus and the pineal gland. Blue dotted lines indicate sympathetic efferences to the heart and thoracic organs. Green lines indicate sympathetic afferences to the sympathetic ganglionic chain and from it to the adrenal glands. In the right side of $C$ spinal roots are shown, while in the left side only the sympathetic ganglionic chain is shown.

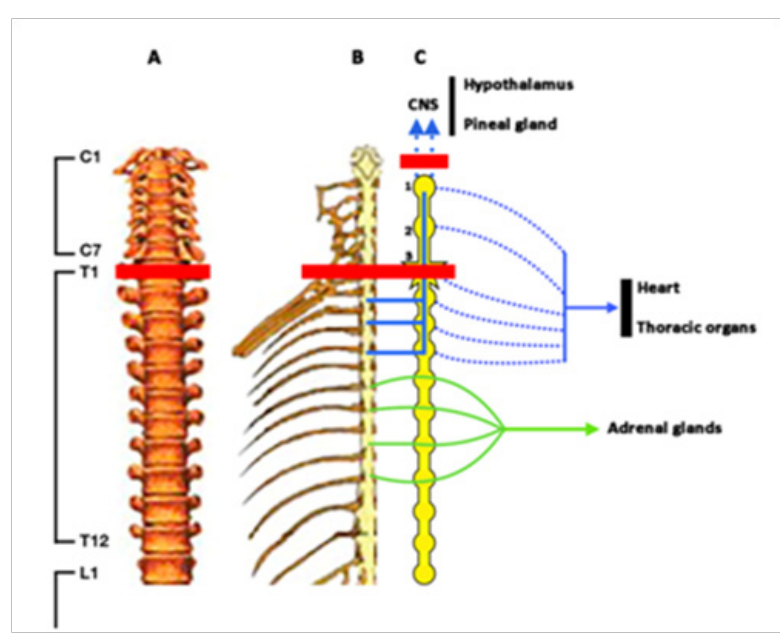

Figure 2 Legend is the same than in Figure I, but in this case the red line indicates the consequences of a complete lesion of the spine at TI level. Loss of afferences from TI lead to the loss of efferent sympathetic signals to the hypothalamus and the pineal gland, and partially to the heart and thoracic organs. The result is a decrease in hypothalamic release of catecholamines resulting in deficient $\mathrm{GH}$ secretion, and the loss of the adrenergic control of the pineal gland which results in the absence of melatonin production. Orthostatic hypotension also appears in this case as a consequence of the decreased sympathetic efferences to the cardiovascular system.

A deficient or insufficient GH secretion in SCI could explain perfectly the metabolic abnormalities seen in these patients, as described in the Introduction, and contribute to the development of osteopenia and osteoporosis. Moreover, in our study ${ }^{9}$ we found that plasma levels of IGF-I were in low values in these SCI patients. As we recently reported ${ }^{26}$ liver production of IGF-I is not fully dependent on $\mathrm{GH}$, but it is well known that the administration of the hormone or its normal secretion induces the expression of IGF-I in liver and many other organs and tissues, including the brain. Therefore, an analysis of GH secretion in response to specific stimuli should be mandatory in tetraplegic patients and a treatment with the hormone should be scheduled if GH-deficiency exists. Apart of these, there are several studies indicating that GH may play a key role in the spinal cord, both during the fetal life and in adulthood. ${ }^{27-29}$ For a more detailed explanation on the effects of $\mathrm{GH}$ on spinal cord and nerve repair see reference. ${ }^{30}$

All these reasons led us to use $\mathrm{GH}$ administration inside the protocols for treating SCI patients in the Medical Center Foltra. For the same reasons that in this review we postulate that there is a deficient (or insufficient) GH secretion in tetraplegic patients, we affirm that the pineal production of MT must also be affected in SCI; mainly in SCI patients with upper cervical injuries. MT levels in human serum and urine rise during the daily dark period. ${ }^{31}$ The pineal production of MT in humans is a process of photo-transduction stimulated by darkness. The natural decrease in light signals to the eye, occurring while night begins, leads to the production of nervous signals sent through the retinohypothalamic tract to the suprachiasmatic nucleus, and from this to the spinal cord that sends afferent signals to the superior cervical ganglion (Figure 1) in which these signals induce a release of catecholamines that reach the pineal gland where they promote the synthesis and release of MT. Therefore, the loss, or decrease, of these adrenergic signals to the pineal gland must affect the production of MT. In fact, superior cervical ganglionectomy in rats induces early changes in $24 \mathrm{~h}$ rhythms and in hypothalamic content of noradrenaline. ${ }^{32}$

Foryears it has been considered that MT simply acted as a 
synchronizer of the sleep-wake rhythm. This is the reason by which small doses of this indolamine are usually prescribed to subjects in which physiologically MT secretion did not occur (children under 2-3years or elderly) or to resynchronize the organism avoiding jetlag. However, we currently know that MT accomplishes many others and even more important functions in the organism. For instance, it is a highly effective hormone for reducing oxidative stress, via direct detoxification of reactive oxygen and reactive nitrogen species, stimulates antioxidant enzymes and suppress the activity of pro-oxidant enzymes, chelates several transition metals. ${ }^{33}$ MT is also an important neuroprotective hormone reducing the effects of hemorrhagic stroke in several experimental models, acting as an antioxidant, anti-apoptosis and anti-inflammatory agent. ${ }^{34}$ MT plays an important role as an agent protective of mitochondria and regulates the electron transport chain responsible of the mitochondrial production of energy. ${ }^{35}$ In addition, recent reports describe the therapeutic effects of MT on gastrointestinal protection, psychiatric and neurodegenerative pathologies, cardiovascular diseases and oncostatic effects. ${ }^{36,37}$

As previously reported by Zeitzer et al ${ }^{17}$ MT has a positive effect in humans. Accordingly, we will analyze what happens in tetraplegic patients in which presumably exists deficient MT production. Curiously few studies related a deficiency or loss of MT secretion with the numerous morbidities associated to SCI injuries, with the exception of sleep disorders. In the first of these studies ${ }^{38}$ it was described that patients with cervical SCI in which there was not nocturnal MT secretion, sleep efficiency was significantly lower than in patients with thoracic SCI and normal MT rhythms; moreover, cervical SCI patients showed significantly increased REM-onset latency as compared to patients with thoracic SCI. Moreover, the diminished sleep in cervical SCI was not due to sleep apnea or any other known reason. These findings were confirmed in a more recent study $^{39}$ in which a complete absence of nocturnal MT secretion was observed in tetraplegic patients leading to a poor sleep quality. These authors also found decreased sleep quality in paraplegic patients, despite that MT secretion in this group was similar to that in controls. Another study concluded that sleep disturbances are more frequent in SCI patients than in the normal population, ${ }^{40}$ a finding that was more exhaustively studied recently. ${ }^{41}$ These authors indicated that $92 \%$ of the SCI patients studied had poor sleep quality and $96 \%$ of them showed daytime fatigue, both more marked in cervical SCI patients. These findings have been attributed to breathing problems rather than to an impaired MT secretion. Similar conclusions have been reported in other studies in which the poor sleep efficiency has been related to sleep apneas and neuromuscular weakness that negatively affects respiratory function. ${ }^{41-43}$ In any case, people with tetraplegia suffer sleep problems and poor quality of life $;{ }^{44}$ moreover, these problems associated to the increased metabolic disorders (as described in the Introduction) place them in an increased risk for suffering stroke or myocardial infarction. Despite that a pilot clinical trial, ${ }^{45}$ carried out in eight patients with tetraplegia and absence of MT production, in which sleep disorders existed, showed that treating them with $8 \mathrm{mg}$ of the MT agonist Ramelteon did not produce significant effects on the existing sleep problems, we think that the reported multiple beneficial effects of MT, and the absence of any adverse effect that it might produce, make this hormone useful when treating SCI patients. This is the reason by which we administered oral MT (at doses no lesser than $50 \mathrm{mg} /$ day) to any SCI patient being treated in the Medical Center Foltra.

No data exist indicating that SCI patients can develop an exaggerated response to an allergen. However, complete SCI above
T8 (Figure 1) can present a deficient response of hypothalamic and adrenal catecholamines and adrenal corticosteroids (stimulated by adrenaline via an ACTH-independent pathway), the natural inhibitors of the response to enhanced histamine release that can lead to an anaphylactic shock and even to death. A pilot study in SCI adults suggests that repeated exposure to natural rubber latex products because of long-term indwelling catheter usage may be at risk for the development of allergy to latex ${ }^{46}$ However, a following study suggested that adult patients with chronic neurological defects resulting from SCI showed a low risk of latex sensitization, something that contrasts with the high level of sensitization observed in children with spina bifida in the same study. ${ }^{47}$

Here we will describe a case of an intense allergic reaction observed in a young girl with complete SCI at C4-C5 level, most likely produced by a colorant present in an ice cream.

\section{Case report}

The patient was a 6year-old girl who came to our Medical Center to be treated for a SCI C4-C5 (AIS A) occurred 1 year before after a traffic accident. The patient was originally from a South American country where she had never eaten that type of food coloring. Two hours after eating that ice cream her mother noticed the appearance of red spots on her face, but as it was bedtime she did not give too much importance to it. The next day the mother observed that these red spots had grown in size and were widely distributed throughout the body, then she called us (it was Sunday) to examine the girl. When examined, we observed many large papulo-macular and erythematous lesions on the face, back, arms and legs (Figure 3). Her eyelids were swollen but there were no signs of glottis edema or breathing problems. We administered her an antihistamine, Cetirizine dihydrochloride (Zyrtec, UCB Pharma, Madrid, Spain), 5mg every $12 \mathrm{~h}$, and soon the problem was solved. Since the patient did not notice any itching, because of her complete sensitive and motor SCI, her mother did not think of an allergic reaction. We cannot know how this reaction could have progressed if we did not interrupt it with the antihistamine drug. After knowing its progressive and intense evolution, it is likely that very important consequences might have appeared because of the absence of physiological inhibitors of the allergic reaction due to her SCI. At this point is important to remark that the patient was taking melatonin (50mg/day), an anti-inflammatory hormone effective to improve disease severity in children with atopic dermatitis, ${ }^{48}$ and perhaps it contributed to diminish the severity of the clinical process.

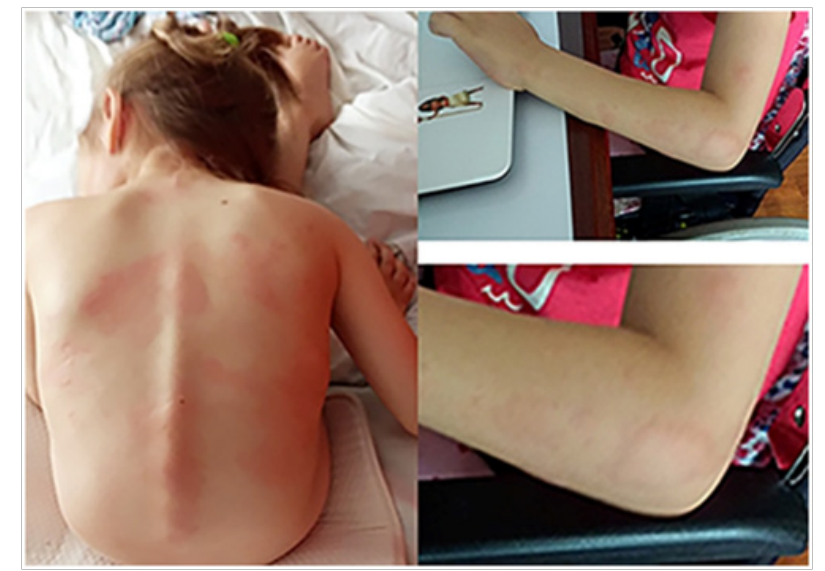

Figure 3 Allergic reaction in a C4-C5 tetraplegic girl. Pictures were taken I 4 hours after the allergic reaction commenced. Note the wide distribution of the papulo-macular and erythematous lesions and the intensity of them before being treated with an antihistamine drug. 


\section{Conclusion}

Data from this review and our own experience allow us to conclude that a GH secretion study should be carried out in every tetraplegic patient, and that $\mathrm{GH}$ administration is useful and safe for treating tetraplegia and preventing or correcting a number of morbidities associated to this pathology. The same occurs with melatonin that most likely is not produced in tetraplegics due to the loss of sympathetic inputs to the pineal gland. Melatonin is also very safe and useful not only for correcting sleep disorders in SCI, but also because of its several positive effects. The possibility of exacerbated allergic reactions should be studied in tetraplegic patients, because of the loss or decreased production of natural inhibitors of histamine in them.

\section{Acknowledgements}

This study has been funded by Foundation Foltra (Teo, Spain), Grant n ${ }^{\circ} 2017 / 7$.

\section{Conflict of interest}

Author declares that there is no conflict of interest.

\section{References}

1. Harvey LA. Physiotherapy rehabilitation for people with spinal cord injuries. J Physiother. 2016;62(1):4-11.

2. World Health Organization. 2013.

3. Bauman WA, Spungen AM, Zhong YG, et al. Depressed serum high density lipoprotein colesterol levels in veterans with spinal cord injury. Paraplegia. 1992;30(10):697-703.

4. Zlotolow SP, Levy E, Bauman WA. The serum lipoprotein profiles in veterans with paraplegia: the relationships to nutritional factors and body mass index. J Am Paraplegia Soc. 1992;15(3):158-162.

5. Bauman WA, Spungen AM. Carbohydrate and lipid metabolism in chronic spinal cord injury. J Spinal Cord Med. 2001;24(4):266-277.

6. Gilbert O, Croffoot JR, Taylor AJ, et al. Serum lipid concentrations among persons with spinal cord injury-a systematic review and metaanalysis of the literature. Atherosclerosis. 2014;232(2):305-312.

7. Laclaustra M, Van Den Berg EL, Hurtado Roca Y, et al. Serum lipid profile in subjects with traumatic spinal cord injury. PLoSOne. 2015;10(2):e0115522.

8. Köseoglu BF, Safer VB, Öken Ö, et al. Cardiovascular disease risk in people with spinal cord injury: is there a possible association between reduced lung function and increased risk of diabetes and hypertension? Spinal Cord. 2017;55(1):87-93.

9. Sanz E. Acciones de la hormona de crecimiento sobre el metabolismo óseo. Spain: Doctoral thesis. University of Santiago de Compostela; 2015.

10. Shoenfeld Y, Shapiro Y, Ohry A, et al. Orthostatic hypotension in amputees and subjects with spinal cord injuries. Arch Phys Med Rehabil. 1978;59(3):138-141.

11. Claydon VE, Krassioukov AV. Orthostatic hypotension and autonomic pathways after spinal cord injury. $J$ Neurotrauma. 2006;23(12):1713-1725.

12. Wecht JM, Bauman WA. Implication of altered autonomic control for orthostatic tolerance in SCI. Auton Neurosci. 2017.

13. Shetty KR, Sutton CH, Mattson DE, et al. Hyposomatomedinemia in quadriplegic men. Am J Med Sci. 1993;305(2):95-100.
14. Bauman WA, Spungen AM, Flanagan S, et al. Blunted growth hormone response to intravenous arginine in subjects with a spinal cord injury. Horm Metab Res. 1994;26(3):152-156.

15. Devesa J, Lima L, Tresguerres JA. Neuroendocrine control of growth hormone secretion in humans. Trends Endocrinol Metab. 1992;3(5):175-183.

16. Li Y, Jiang DH, Wang ML, et al. Rhythms of serum melatonin in patients with spinal lesions at the cervical, thoracic or lumbar region. Clin Endocrinol (Oxf). 1989;30(1):47-56.

17. Zeitzer JM, Ayas NT, Shea SA, et al. Absence of detectable melatonin and preservation of cortisol and thyrotropinrhythms in tetraplegia. J Clin Endocrinol Metab. 2000;85(6):2189-2196.

18. Borges R. Histamine $\mathrm{H} 1$ receptor activation mediates the preferential release of adrenaline in the rat adrenal gland. Life Sci. 1994;54(9):631-640.

19. Bealer SL, Crowley WR. Stimulation of central and systemic oxytocin release by histamine in the paraventricular hypothalamic nucelus: evidence for an interaction with norepinephrine. Endocrinology. 1999;140(3):1158-1164

20. Devesa J, Arce V, Lois N, et al. Alpha 2-adrenergic agonism enhances the growth hormone $(\mathrm{GH})$ response to $\mathrm{GH}$-releasing hormone through an inhibition of hypothalamic somatostatin release in normal men. $J$ Clin Endocrinol Metab. 1990;71(6):1581-1588.

21. Arce V, Cella SG, Loche S, et al. Synergistic effect of growth hormonereleasing hormone (GHRH) and clonidine in stimulating $\mathrm{GH}$ release in young and old dogs. Brain Res. 1990;537(1-2):359-362.

22. Devesa J, Díaz MJ, Tresguerres JA, et al. Evidence that alpha 2 -adrenergic pathways play a major role in growth hormone $(\mathrm{GH})$ neuroregulation: alpha 2-adrenergic agonism counteracts the inhibitory effect of muscarinic cholinergic receptor blockade on the GH response to GH-releasing hormone, while alpha 2-adrenergic blockade diminishes the potentiating effect of increased cholinergic to neon such stimulation in normal men. $J$ Clin Endocrinol Metab. 1991;73(2):251-256.

23. Lima L, Arce V, Díaz MJ, et al. Clonidine pretreatment modifies the growth hormone secretory pattern induced by short-term continuous GRF infusion in normal man. Clin Endo crinol (Oxf). 1991;35(2):129-135.

24. Lima L, Arce V, Tresguerres JA, et al. Studies on alpha 2-adrenergic modulation of hypothalamic somatostatin secretion in rats. Life Sci. 1993;53(8):665-668.

25. Lima L, Arce V, Díaz MJ, et al. Glucocorticoids may inhibit growth hormone release by enhancing beta-adrenergic responsiveness in hypothalamic somatostatin neurons. $J$ Clin Endocrinol Metab. 1993;76(2):439-444.

26. Devesa J, Almengló C, Devesa P. Multiple effects of growth hormone in the body: Is it really the hormone for growth. Clin Med Insights Endocrinol Diabetes. 2016;9:47-71.

27. McIlwain DL, Hoke VB, Kopchick JJ, et al. Differential inhibition of postnatal brain, spinal cord and body growth by a growth hormone antagonist. BMC Neurosci. 2004;5:6-9.

28. Chen L, Lund PK, Burgess SB, et al. Growth hormone, insulin-like growth factor I, and motoneuron size. J Neurobiol. 1997;32(2):202-212.

29. Koohestani F, Brown CM, Meisami E. Postnatal growth hormone deficiency in growing rats causes marked decline in the activity of spinal cord acetylcholinesterase but not butyryl cholinesterase. Int J Dev Neurosci. 2012;30(7):578-583

30. Devesa J, Alonso A, López N, et al. Growth hormone (GH) and rehabilitation promoted distal innervation in a child affected by caudal regression syndrome. Int J Mol Sci. 2017;18(1). 
31. Wurtman RJ, Ozaki Y. Physiological control of melatonin synthesis and secretion: mechanisms, generating rhythms in melatonin, methoxytryptophol, and arginine vasotocin levels and effects on the pineal of endogenous catecholamines, the estrous cycle, and environmental lighting. J Neural Transm. 1978;Suppl(13):59-70.

32. Castrillón PO, Cardinali DP, Pazo D, et al. Effect of superior cervical ganglionectomyon 24-h variations in hormone secretion from the anterior hypophysis and in hypothalamic mono amine turn over during the preclinical phase of Freund's adjuvant arthritis in rats. $J$ Neuro endocrinol. 2001;13(3):288-295.

33. Reiter RJ, Mayo JC, Tan DX, et al. Melatonin as an antioxidant: under promises but over delivers. J Pineal Res. 2016;61(3):253-278.

34. Dong Y, Fan C, HuW, et al. Melatonin attenuated early brain injury induced by sub arachnoid hemorrhage via regulating NLRP3 inflammasome and apoptosis signaling. J Pineal Res. 2016;60(3):253-262.

35. Acuña Castroviejo D, Escames G, León J, et al. Mitochondrial regulation by melatonin and its metabolites. Av Exp Med Biol. 2003;527:549-557.

36. Tordjman S, Chokron S, Delorme R, et al. Melatonin: pharmacology, functions and therapeutic benefits. Cuur Neuro pharmacol. 2017;15(3):434-443.

37. Reiter RJ, Rosales Corral SA, Tan DX, et al. Melatonin, a full service anti-cancer agent: inhibition of initiation, progression and metastasis. Int J Mol Sci. 2017;18(4)

38. Scheer FA, Zeitzer JM, Ayas NT, et al. Reduced sleep efficiency in cervical spinal cord injury; association with abolished night time melatonin secretion. Spinal Cord. 2006;44(2):78-81.
39. Verheggen RJ, Jones H, Nyakayiru J, et al. Complete absence of evening melatonin increase in tetraplegics. FASEB J. 2012;26(7):3059-3064.

40. Giannoccaro MP, Moghadam KK, Pizza F, et al. Sleep disorders in patients with spinal cord injury. Sleep Med Rev. 2013;17(6):399-409.

41. Sankari A, Bascom A, Oomman S, et al. Sleep disordered breathing in chronic spinal cord injury. J Clin Sleep Med. 2014;10(1):65-72.

42. Chiodo AE, Sitrin RG, Bauman KA. Sleep disordered breathing in spinal cord injury: a systematic review. J Spinal Cord Med. 2016;39(4):374-382.

43. Schembri R, Spong J, Graco M, et al. Neuropsychological function in patients with acute tetraplegia and sleep disordered breathing. Sleep. 2016;40(2).

44. Spong J, Graco M, Brown DJ, et al. Subjective sleep disturbances and quality of life in chronic tetraplegia. Spinal Cord. 2015;53(8):636-640.

45. Zeitzer JM, Ku B, Ota D, et al. Randomized controlled trial of pharmacological replacement of melatonin for sleep disruption in individuals with tetraplegia. J Spinal Cord Med. 2014;37(1):46-53.

46. Monasterio EA, Barber DB, Rogers SJ, et al. Latex allergy in adults with spinal cord injury: a pilot investigation. J Spinal Cord Med. 2000;23(1):6-9.

47. Mertes PM, Mouton C, Fremont S, et al. Latex hypersensitivity in spinal cord injured adult patients. Anaesth Intensive Care. 2001;29(4):393-399.

48. Chang YS, Lin MH, Lee JH, et al. Melatonin supplementation for children with a topic dermatitis and sleep disturbance: a randomized clinical trial. JAMA Pediatr. 2016;170(1):35-42. 